\title{
Model and Integrate Medical Resource Available Times and Relationships in Verifiably Correct Executable Medical Best Practice Guideline Models (Extended Version)
}

\author{
Chunhui Guo, Zhicheng Fu, Zhenyu Zhang, Shangping Ren \\ Department of Computer Science \\ Illinois Institute of Technology \\ Chicago, IL 60616, USA \\ \{cguo13, zfu11, zzhang111\}@hawk.iit.edu, ren@iit.edu
}

\author{
Lui Sha \\ Department of Computer Science \\ University of Illinois at Urbana-Champaign \\ Urbana, IL 61801, USA \\ 1rs@illinois.edu
}

\begin{abstract}
Improving patient care safety is an ultimate objective for medical cyber-physical systems. A recent study shows that the patients' death rate is significantly reduced by computerizing medical best practice guidelines [21]. Recent data also show that some morbidity and mortality in emergency care are directly caused by delayed or interrupted treatment due to lack of medical resources [20]. However, medical guidelines usually do not provide guidance on medical resource demands and how to manage potential unexpected delays in resource availability. If medical resources are temporarily unavailable, safety properties in existing executable medical guideline models may fail which may cause increased risk to patients under care.

The paper presents a separately model and jointly verify (SMJV) architecture to separately model medical resource available times and relationships and jointly verify safety properties of existing medical best practice guideline models with resource models being integrated in. The SMJV architecture allows medical staff to effectively manage medical resource demands and unexpected resource availability delays during emergency care. The separated modeling approach also allows different domain professionals to make independent model modifications, facilitates the management of frequent resource availability changes, and enables resource statechart reuse in multiple medical guideline models. A simplified stroke scenario is used as a case study to investigate the effectiveness and validity of the SMJV architecture. The case study indicates that the SMJV architecture is able to identify unsafe properties caused by unexpected resource delays.
\end{abstract}

\section{INTRODUCTION}

Patient care safety is time critical, especially for emergency care. Improving patient care safety needs to consider dynamic patient conditions, proper treatment procedures, treatment timing constraints, medical resource demands, and unexpected resource delays. Due to the complicated interdependencies among patient conditions, treatments and their corresponding timing constraints, and needed medical resources, it is difficult for medical professionals to consider and process all the dynamic information and make quick and accurate decisions. Hence, a computer system is needed to assist

This work is supported in part by NSF CNS 1545008 and NSF CNS 1545002. medical professionals in validating and verifying the medical guideline models with all the information being taken into considerations.

Medical best practice guidelines are intended to optimize patient care, that are informed by a systematic review of evidence and an assessment of the benefits and harms of alternative care options. For an acute illness, there is often a golden time window during which prompt and proper medical treatments have the highest likelihood for optimal patient care outcomes [22]. As patient condition can change rapidly, delayed or interrupted medical treatment procedures will often result in increased morbidity and mortality. Doctors and researchers at the Ottawa Hospital Research Institute studied 15,160 patients waiting for emergency medical procedures and found that of those 2820 patients who experienced delays, 138 died due to lack of timely medical care, such as delay due to lack of operating room resources and medical devices [20]. We use a stoke patient as an example to show the complex interdependencies among patient condition, proper treatments and their corresponding golden time, and needed medical resources depicted in Fig. 1.

Stroke Scenario: An ischemic stroke occurs when a clot or a mass blocks a blood vessel cutting off blood flow to a part of the brain and results in a corresponding loss of neurological function [2]. The intravenous (IV) tissue plasminogen activator (tPA) injection is a standard treatment for ischemic stroke patients and it is most effective during the initial 3-hour window from the onset of stroke symptoms [3]. The treatment window can be extended from 3 to 4.5 hours for certain patients, but the risks are increased [17]. Some patients can be treated by dripping tPA directly on the clot through a microcatheter within 6 hours from the onset of stroke symptoms [26]. However, the micro-catheter tPA treatment requires specialists to control tPA dose, special equipments to put the microcatheter into blood vessels, and technicians to operate the special equipment.

In addition, in order to use the tPA treatment, we must 
ensure that (1) CT scan does not show hemorrhage, and (2) the patient's blood pressure is under control. To derive the conclusion that the patient does not have hemorrhage, we would need medical resources including a CT machine, a CT technician, and a radiologist. If a patient's blood pressure is not within the range for tPA administration, a specialist is required to control blood pressure.

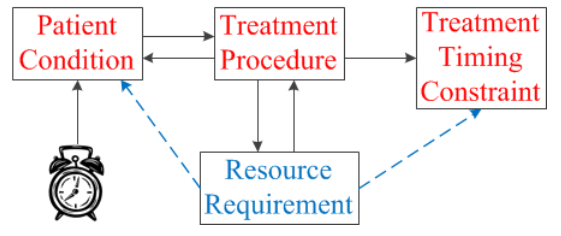

Fig. 1. Interdependencies

As the stroke scenario indicates, for many acute emergency care, taking proper treatment actions is a challenging task. Recent study conducted by Houston Methodist Hospital Research Institute shows that computerized executable medical best practice guidelines can significantly help physicians to make treatment decisions, improve patient care safety, and reduce patients' death rate (for sepsis patients, the death rate drops from $31 \%$ to $14 \%$ by computerizing a sepsis best practice protocol) [21]. For this reason, many computer executable medical best practice guideline models are developed over the past decade to assist medical professionals in medical practices [4], [23], [28], [11], [27].

As medical guideline handbooks often do not provide instructions on medical resource demands and how to manage unexpected delays in resource availability, their computerized models based on the medical handbooks often also lack these information. In elective medical procedures, patient's treatments are scheduled according to resource availability. However, it is not possible to schedule patient treatments in emergency care. If medical resources are temporarily unavailable for emergency care, certain transitions in computerized medical guideline models may be blocked, which can falsify validated/verified safety properties. In such cases, medical staff have to improvise treatment decisions, which increases patient's risk.

Take a stroke patient as an example, assume a stroke patient's onset time is 0 and a physician orders a CT scan for the patient at time 20 (minutes). If the CT machine is available, the tPA administration can be completed within the 3-hour window. However, if the CT machine or a radiologist is not available for 200 minutes, the tPA administration will not be completed within 3-hour time window. Hence, taking considerations of required medical resources' available time in validating and verifying executable medical guideline models is essential for patient care safety.

The resource available time is often given in a timetable [25]. Medical resources by themselves often do not have any relationships, but when they are associated with medical treatment procedures, the procedures may require certain relationships among the resources. For the CT scan scenario in the stroke example, obtaining CT results contains two main sequential steps, i.e., taking CT image followed by image diagnosis. The imaging step requires both a CT machine and a CT technician at the same time; while a radiologist is needed to diagnose images after the image is obtained. Hence, the relationships between machines and technicians and between machines/technicians and radiologists are concurrent and sequential, respectively.

It is worth pointing out that the study of temporarily unavailable medical resources is a challenging medical cyberphysical problem. The interruption to a medical procedure is different from interruptions handling in computer science. The medical resource available time and their relationship issue has to be addressed in a systematic way and in conjunction with consideration of dynamic patient conditions, medical treatment procedures, and treatment timing constraints. It involves domain knowledge from three areas: (1) medical resource available time which is usually managed by medical schedulers, (2) illness and treatments which is usually managed by medical doctors; and (3) safety verification of computerized models done by computer scientists.

The paper presents a separately model and jointly verify (SMJV) architecture to separately model medical resource available times and relationships and jointly verify safety properties of existing medical best practice guideline models with resource models being integrated in. This architecture is built on top of an existing framework which transforms validated medical best practice guideline statechart models to UPPAAL time automata with Y2U tool [12] so that the medical guidelines' safety properties can be formally verified. Fig. 2 depicts the high level architecture of our approach. As depicted in Fig. 2, the SMJV architecture allows different personals to focus on their own knowledge domains and make independent model modifications when needed. It requires minimal change to existing medical best practice guideline models, and can directly apply existing work/tools to address patient care safety under dynamic patient conditions by exploring different execution paths in the medical best practice guideline models with resource demands. In addition, the separated medical resource models built with the SMJV architecture can be reused by multiple medical guideline statecharts and requires minimal modification caused by resource demands and available time changes.

To our best knowledge, the SMJV architecture is the first computerized formal verification architecture to automatically assist medical staff to effectively manage medical resource demands and unexpected resource availability delays during emergency care. Without a computerized system, engineers need to be involved in clinical care to manually modify and verify medical guideline models when resource demands and available times change. Furthermore, the manual modifications are error prone, which increases patients' risk.

The rest of the paper is organized as follows. We discuss related work in Section II. Section III introduces a framework for building verifiably correct executable medical guideline models. We identify basic medical relationships, define med- 


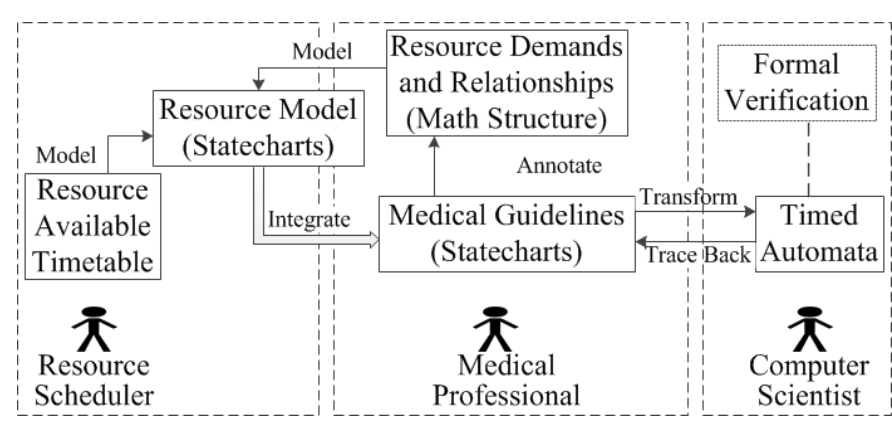

Fig. 2. SMJV Architecture

ical resource demands, and automatically annotate resource demands in verifiably correct executable medical guidelines in Section IV. We present the approaches to model medical resource demands and relationships and resource available times in Section V and Section VI, respectively. Section VII defines the procedure for integrating medical resource models into existing medical guideline statecharts. A simplified stroke case study is given in Section VIII to illustrate the effectiveness and advantages of the presented SMJV architecture. We draw conclusions in Section IX.

\section{RELATED WORK}

Over the past two decades, significant amount of efforts have been made in obtaining various computer-interpretable models and developing tools for the management of medical guidelines, such as GLIF [23], Asbru [4], EON [28], GLARE [27], and PROforma [11], to name a few. To facilitate easy clinical validation, Wu et al. have developed a workflow adaptation approach [31] to help physicians safely adapt workflows to react to patient adverse events and a treatment validation protocol [30] to enforce the correct execution sequence of performing a treatment based on precondition validations, side effects monitoring, and expected responses checking. In addition, our previous work [12] also designed a platform to model medical guidelines with statecharts [15] and automatically transform statecharts to timed automata [1] for formal verification. These models and tools integrated clinical guidelines with the treatment flow and provided patientspecific advice when and where needed. These tools provide a better chance of positively impacting clinician behaviors by reducing unjustified practice variations. However, the effects and impacts of medical treatment procedure delays due to temporarily unavailable medical resources have not been well addressed.

Research on patients and resource scheduling is well established and growing. Many good medical resource scheduling systems have been developed to reduce patient waiting times and also improve the utilization of critical resources by means of tracking the availability of resources, projecting future demands for service and automating the assignment of resources to needs [19], [25], [29], [9], [14]. For instance, the problem of scheduling patients in CT scanning department for improving resource utilization is addressed in [24]. A comprehensive survey of the research is provide by [6] and [13]. Though scheduling software is destined for growth, integrating medical resource availabilities generated by scheduling systems with executable medical best practice guideline models to improve safety of treatments is yet to be addressed.

To study the medical resource availability issue in existing medical guideline models, Kim and Lee et al. [18] used timed and resource-oriented statecharts and took a direct modification approach by specifying required resource information in transition guards and as state constraints to analyze the time and resource-constrained behavior of the system. With similar ideas, Christov and Lori et al. [7], [8] used Little-JIL to model the processes in medical guidelines and represented resource as preconditions of process steps. All these efforts have shown that medical resource availabilities is critical to the safety of executable medical best practice guideline models. In this paper, we present an approach that separately models resource available times and their relationships and jointly verify the safety properties of statechart-based computerized medical best practice guideline models with resource models being integrated in.

\section{Verifiably Correct Executable Medical Best PRACTICE GUIDELINES}

Our previous work [12] designed a platform to build verifiably correct executable medical guidelines. In particular, we use statecharts [15] to model medical guidelines and interact with medical professionals to validate the correctness of the medical guideline models. The statecharts built with Yakindu tool [16] are then automatically transformed to UPPAAL timed automata [5] by the developed Y2U tool [12], so that the safety properties required by the model can be formally verified with UPPAAL. We use the simplified stroke scenario presented in Section I as an example to briefly summarize the process of building verifiably correct executable medical guideline models.

We use Yakindu statecharts to model the stroke treatment guideline given in [17]. For illustration and easy understanding purpose, we show a simplified stroke statechart model in Fig. 3, which only focuses on the CT scan and TPA administration procedures and omits other medical procedure details. In the simplified statechart shown in Fig. 3, we assume that upon patient arrival, treatments to control blood pressure have been immediately performed and the patient blood pressure is quickly brought within the safe range if this can be done.

In the statechart shown in Fig. 3, two medical procedures CTscan and givetPA are modeled by Yakindu statechart as events. In Yakindu statecharts, events can be raised by both states and transitions. For instance, the entry action of state CT (entry/ raise CTscan) raises event CTscan when state CT is entered. The event givetPA is raised by the transition from state tPAcheck to state tPA if tPA is administrated (the value of boolean variable tPAad is true). In the simplified stroke statechart model (Fig. 3), the two time related variables curT and onsetT represent the current system time and the onset time of stroke symptoms, respectively. We assume that the 


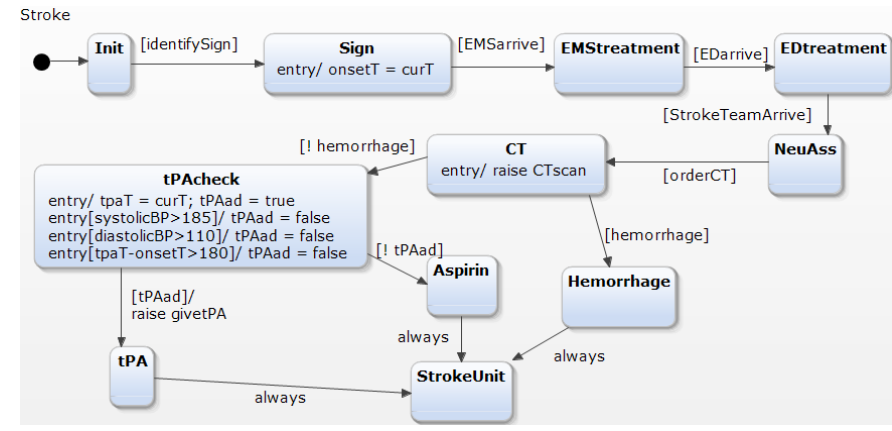

Fig. 3. Simplified Stroke Yakindu Statechart Model

time unit in the simplified stroke statechart model is minute. Hence, the remaining time of the 3-hour tPA treatment window can be calculated by formula $180-(\operatorname{curT}-$ onsetT).

The simplified stroke statechart model in Fig. 3 is transformed to UPPAAL time automata as shown in Fig. 4 with the Y2U tool [12]. There are safety properties in the simplified stroke scenario, i.e., P1: the tPA is injected only if a CT scan shows no hemorrhage and systolic and diastolic blood pressures are smaller than or equal to $185 \mathrm{~mm} \mathrm{Hg}$ and 110 $\mathrm{mm} \mathrm{Hg}$; and P2: the tPA administration is completed within 3 hours from onset of symptoms. The safety properties $\mathbf{P 1}$ and $\mathbf{P 2}$ are verified in UPPAAL by formula (1) and formula (2), respectively.

$$
\begin{array}{r}
A[\text { ] Stroke.tPA imply systolicBP }<=185 \& \& \\
\text { diastolicBP }<=110 \& \& \text { ! hemorrhage }
\end{array}
$$

$$
A[\text { ] Stroke.tPAcheck imply tpaT }- \text { onsetT }<=180
$$

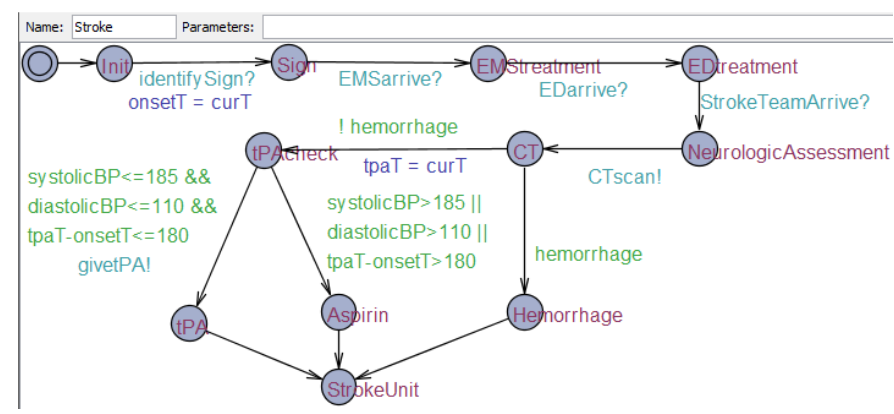

Fig. 4. Simplified Stroke UPPAAL Model

If all the required medical resources are available, both clinical validation results of stroke Yakindu model in Fig. 3 and formal verification results of stroke UPPAAL model in Fig. 4 show that both properties $\mathbf{P} 1$ and $\mathbf{P} 2$ are satisfied. However, if both CT machines and CT technicians are available after 200 minutes from onset of the symptoms, the stroke statechart is then blocked at state CT for 200 minutes. In this scenario, the safety property $\mathbf{P} 2$ fails.

The example reveals a fact that safety properties validated and verified in medical guideline models based on the assumption that medical resources are available can fail because of temporarily unavailable resources. Hence, taking into consideration of medical resource available times and relationships in developing verifiable medical guideline models is essential in ensuring patient care safety. The paper addresses the issue by integrating medical resource available times and relationships with executable medical guideline models. In particular, in the next two sections, we first define medical resource demands with resource relationships and automatically annotate medical resource demands in executable medical guidelines (Section IV), we then model medical resource demands with statecharts (Section V). In Section VI, a mathematical structure is defined to explicitly specify medical resource available times, then the mathematical structure is automatically transformed to statecharts for integration with existing medical guideline statecharts. The medical resource models are then automatically integrated with existing medical guideline statecharts using the boolean variables built in resource statecharts in Section VII. Lastly, we transform the integrated medical guideline statecharts to timed automata [1] by the Y2U tool [12] to formally verify safety properties in the presence of temporarily unavailable medical resources.

\section{Annotate Medical Resource Demands}

To model medical resource demand, we need to identify which resources are required by which medical procedures of a particular medical guideline and resource relationships. In this section, we identify basic medical relationships, define medical resource demand, and automatically annotate medical resource demands in executable medical guidelines.

We use the simplified stroke statechart model shown in Fig. 3 as an example to illustrate medical resource demands of medical guidelines. In the state CT, the request for medical procedure CT scan is modeled as CTscan event in Yakindu statecharts. This event is raised by the entry action of the CT state. According to medical professionals, the CTscan procedure requires CT machines, CT technicians, and radiologists. The relationships between machines and technicians and between machines/technicians and radiologists are concurrent and sequence, respectively. Similarly, the request of giving tPA procedure is modeled as givetPA event which is raised by the action of the transition from state tPAcheck to state tPA. The giving tPA procedure requires tPA fluid and nurses. The examples show that (1) multiple medical resources required by a medical procedure have relationships; (2) the request of medical procedures are modeled as statechart events and can be raised in both states and transitions; and (3) treatment procedure required medical resource demands are not represented in medical guideline models and need to be provided by medical professionals.

\section{A. Medical Resource Relationships}

To represent medical resource demands, we define three basic medical resource relationships as follows:

1) Concurrent Resources are resources required at the same to perform a medical procedure. For example, 
taking CT images requires both $\mathrm{CT}$ machines and CT technicians;

2) Alternative Resources provide multiple resource options for a medical procedure, but only one option is required to perform the procedure. For example, either CT machines or MRI machines can provide brain images;

3) Sequence Resources are required by a medical procedure in time sequence with specified time durations between them. For example, obtaining CT results needs a CT machine and a CT technician to take CT image first, and then requires a radiologist to diagnose images after 10 minutes (assuming it takes 10 minutes to take the CT images).

To formally model medical resource relationships, we define three resource relationship operators and the order of operations as follows.

Definition 1 (Concurrent Resource Operator): The concurrent resource operator $\otimes$ takes two medical resources $r_{1}$ and $r_{2}$ as arguments, i.e., $r_{1} \otimes r_{2}$, and declares that the two medical resources $r_{1}$ and $r_{2}$ are concurrently required.

Definition 2 (Alternative Resource Operator): The alternative resource operator $\oplus$ takes two medical resources $r_{1}$ and $r_{2}$ as arguments, i.e., $r_{1} \oplus r_{2}$, and declares that either medical resource $r_{1}$ or $r_{2}$ is required.

Definition 3 (Sequence Resource Operator): The sequence resource operator $\odot_{t}$ takes two medical resources $r_{1}$ and $r_{2}$ and an integer as arguments, i.e., $r_{1} \odot_{t} r_{2}$, and declares that the medical resource $r_{1}$ is first required and then after $t$ time units resource $r_{2}$ is needed, where $t \geq 0$.

Definition 4 (Operation Order of Resource Relationship Operators): The operation order of the concurrent, alternative, and sequence resource relationship operators is $\otimes>\oplus>$ $\odot t$.

It is not difficult to see that different relationships among medical resources can be represented by the defined three resource relationship operators. We use Example 1 to illustrate how to represent resource relationships with the operators.

Example 1: In the simplified stroke statechart model shown in Fig. 3, a medical procedure CTscan is ordered in state CT. According to medical professionals, the CTscan procedure first requires a CT machine and a CT technician to take CT image and requires a radiologist to diagnose images after 10 minutes. Based on Definition 1, the concurrent relationship between CT machine and CT technician is represented by CT_machine $\otimes$ CT_technician. According to sequence resource operator and operator precedence definitions, i.e., Definition 3 and Definition 4, the sequence relationship between machine/technician and radiologist is represented by

$$
\text { CT_machine } \otimes \text { CT_technician } \odot_{10} \text { radiologist. }
$$

B. Annotate Medical Resource Demands in Executable Medical Guideline Models

Most existing medical guideline models follow guideline handbooks and usually do not provide instructions on medical resource demands and how to manage unexpected delays in resource availability. To represent medical resource demands in executable medical guideline models, we first define medical resource demands and then automatically annotate resource demands in executable medical guideline models.

The resource demand of a given medical procedure specifies the procedure required medical resources and their relationships. We give the formal definition of medical resource demands and an example of the CT scan scenario as follows.

Definition 5 (Resource Demand): The resource demand $d$ of a given medical procedure $p$ is defined as an expression of medical resources $R$ and resource relationship operators $\otimes$, $\oplus$, and $\odot_{t}$, where $R$ is a set of medical resources required by the procedure $p$.

Example 2: As analyzed in Example 1, the CT scan procedure requires three medical resources, i.e., CT machine, CT technician, and radiologist, with relationship given in formula (3). According to Definition 5, the resource demand $d$ of the CT scan procedure is

$$
d=\text { CT_machine } \otimes \text { CT_technician } \odot_{10} \text { radiologist. }
$$

We use the following two steps to automatically annotate medical resources in executable medical guidelines:

1) represent medical resource demands given by medical professionals with a map structure; and

2) automatically annotate resource demands in states and transitions according to the resource demand map and raised medical procedures in corresponding states and transitions.

We define the resource demand map structure as (key, value), where the key is medical procedures that are represented by corresponding event names in the medical guideline statecharts. The value of the resource demand map is the medical resource demand of the corresponding key (medical procedure). We give the formal definition of the resource demand map structure in Definition 6 and show the resource demand map of the simplified stroke scenario in Example 3.

Definition 6: Given an executable medical guideline model $\mathcal{G}$, a set of medical procedures $P=\left\{p_{1}, p_{2}, \ldots, p_{n}\right\}$ in the medical guideline $\mathcal{G}$, and a medical resource demand $d_{i}$ for each medical procedure $p_{i}$, the medical resource demand map $\mathcal{M}_{r}$ is defined as

$$
\begin{aligned}
\mathcal{M}_{r}= & \left\{\left(p_{1}, d_{1}\right),\right. \\
& \left(p_{2}, d_{2}\right), \\
& \ldots \ldots \\
& \left.\left(p_{n}, d_{n}\right)\right\} .
\end{aligned}
$$

Example 3: The simplified stroke statechart model shown in Fig. 3 has two medical procedures CTscan and givetPA. The medical resource demand of the CTscan procedure is given in formula (4). The givetPA procedure requires tPA fluid and a 
nurse. According to Definition 6, the resource demand map of the simplified stroke scenario is

$\left\{\left(\right.\right.$ CTscan, $C T$ machine $\otimes$ CT_technician $\odot_{10}$ radiologist $)$ (givetPA, tPA $\otimes$ nurse) $\}$.

The required demand represented in $\mathcal{M}_{r}$ is independent of executable medical guideline models. With the purpose of not affecting execution behaviors and validation/verification results of medical verifiably correct executable medical guideline models, we annotate medical resource demands by Yakindu statechart comments. The annotation is defined as follows.

Definition 7: Given a state $S$ (or a transition $T$ ) in an executable medical guideline model $\mathcal{G}$, a set of medical procedures $P_{S}=\left\{p_{1}, p_{2}, \ldots, p_{n}\right\}$ modeled in state $S$ (or transition $T$ ), and a medical resource demand map $\mathcal{M}_{r}$ of $\mathcal{G}$, the annotation of state $S$ (or transition $T$ ) is represented as

$$
\text { //@RES : }\left(p_{1}, d_{1}\right),\left(p_{2}, d_{2}\right), \ldots,\left(p_{n}, d_{n}\right) .
$$

Based on the medical resource demand map and the medical resource annotation definitions, we annotate required medical resources in executable medical guideline statecharts with following two steps: first search each state $S$ (and transition $T$ ) in a given medical guideline statechart $\mathcal{G}$; second, if the actions of state $S$ (or transition $T$ ) contain medical procedures in the given medical resource demand map $\mathcal{M}_{r}$, add the annotation, i.e., formula (7), to state $S$ (or transition $T$ ). Algorithm 1 gives the details of the annotation procedure, where the operation $\mathcal{M}_{r}^{\prime}+(p, d)$ in Line 6 inserts the element $(p, d)$ into the map $\mathcal{M}_{r}^{\prime}$.

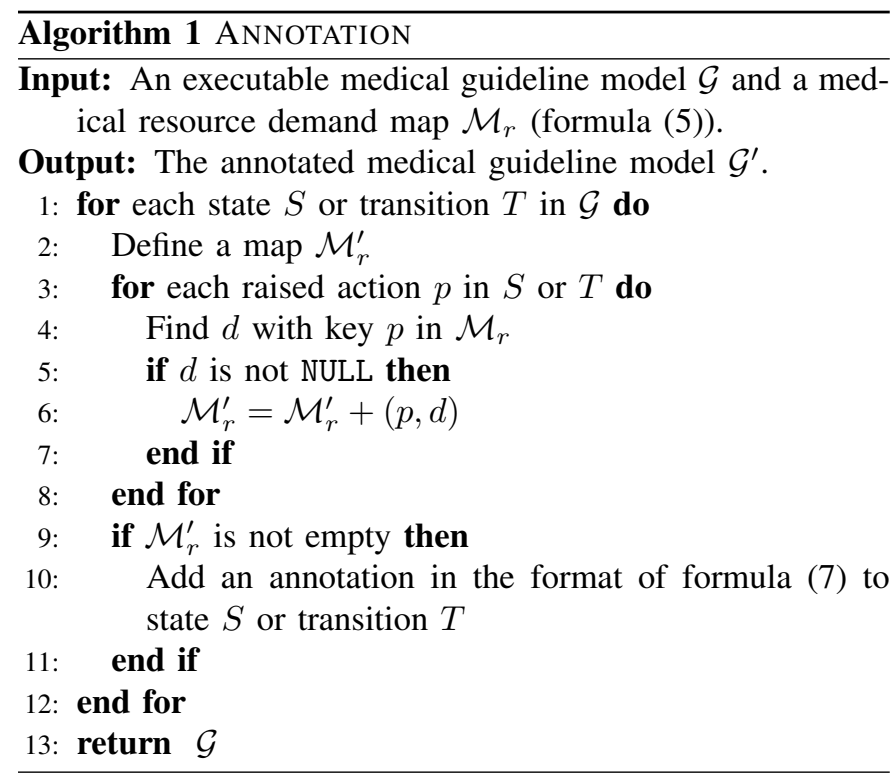

Example 4: Given the simplified stroke statechart model shown in Fig. 3 and a resource demand map of formula (6). The state CT has a medical procedure CTscan. We use CTscan as the key to search the resource demand map given by formula (6) and find resource demand CT_machine $\otimes$ CT_technician $\odot_{10}$ radiologist.
According to Definition 7, we add the annotation "//@RES : (CTscan, CT_machine $\otimes$ CT_technician $\odot_{10}$ radiologist)" to state CT. Similarly, we add the annotation "//@RES : (givetPA, tPA $\otimes$ nurse)" to the transition from state tPAcheck to state tPA. The annotated stroke statechart model by Algorithm 1 is depicted in Fig. $5^{1}$, where the annotated states and transitions are marked by red rectangles.

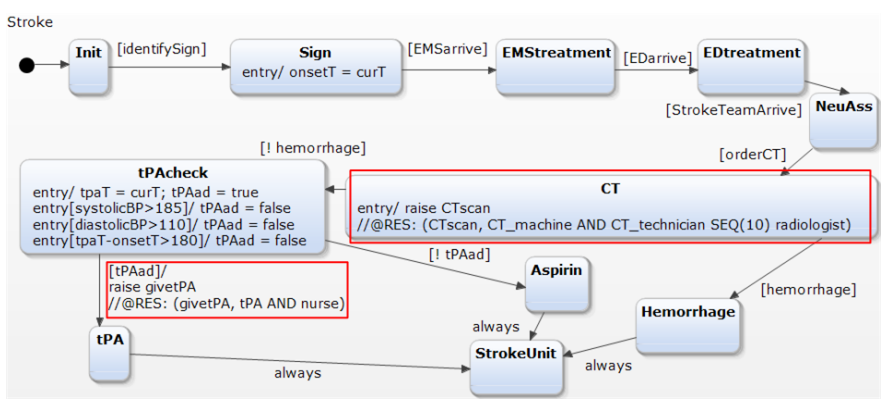

Fig. 5. Annotated Statechart of Simplified Stroke Scenario ${ }^{1}$

\section{Model Medical Resource Demands and Their RELATIONSHIPS}

For a given resource demand map $\mathcal{M}_{r}=\{(p, d)\}$ and its corresponding medical guideline models, we build resource demand statecharts in four steps: (1) design a Timer statechart to record current system time; (2) for each medical resource and each medical procedure in $\mathcal{M}_{r}$, declare a boolean variable to denote whether a resource is available and whether a procedure is able to execute, respectively; (3) design a statechart for each basic resource relationship defined in Section IV-A; and (4) develop a procedure to automatically transform each element in the resource demand map $\mathcal{M}_{r}$ to a statechart by composing the basic resource relationship statecharts.

For the Timer statechart, we use an integer variable curT to denote current system time and let a Timer statechart to increase current time curT. The Timer statechart only contains one state which has a self-loop transition to increase current time curT by 1 every one time unit. Fig. 6 depicts a Timer statechart with time unit as minute, which increases curT by 1 every 60s.

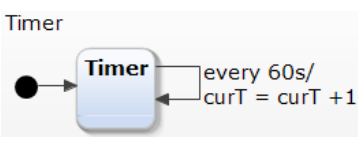

Fig. 6. Timer Statechart

To represent resource demand related variables, we declare an interface named RES. For each unique resource $r$ in resource demands $d$ of a given resource map $\mathcal{M}_{r}$, we declare a boolean variable $V_{r}$ in the interface RES to denote whether the resource $r$ is available at current system time. The variable $V_{r}$ has the same name as the corresponding resource $r$ and the default value is false indicating the resource $r$ is not available initially. The details on how to check whether a resource is

\footnotetext{
${ }^{1}$ In Yakindu statecharts, we use "AND", "OR", and "SEQ $(t)$ " to replace $\otimes, \oplus$, and $\odot_{t}$ in the resource demand annotations, respectively.
} 
available under given resource schedules will be presented in Section VI.

For each medical procedure $p$ in the given resource map $\mathcal{M}_{r}$, we declare a boolean variable $V_{p}$ in the interface to denote whether the procedure $p$ is able to execute from resource demands perspective. Similarly, the variable $V_{p}$ has the same name as the corresponding procedure $p$ (event name) and the default value is false meaning that the procedure $p$ can not be executed initially. The procedure variables are used as anchors for integrating resource demand statecharts with existing medical guideline models in Section VII. We use Example 5 to show the declared variable of the simplified stroke scenario.

Example 5: The medical resource demand map of the simplified stroke scenario is given in formula (6). The resource map contains two medical procedures, i.e., CTscan and givetPA, and five medical resources, i.e., CT_machine, CT_technician, radiologist, tPA, and nurse. The declared resource and procedure variables are shown in Fig. 7.

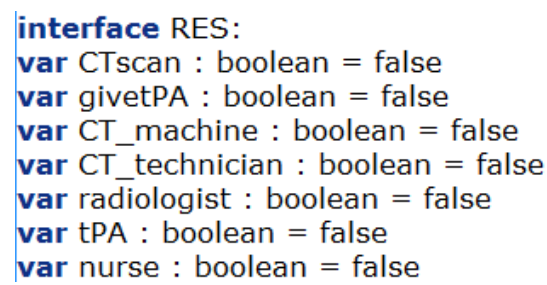

Fig. 7. Resource and Procedure Variables

According to Definition 5, resource demands are represented as compositions of basic resource relationships. To model resource demands with statecharts, we first design a statechart to represent each basic resource relationship defined in Section IV-A, then develop a procedure to automatically transform each resource demand in $\mathcal{M}_{r}$ to a statechart by composing the basic resource relationship statecharts.

Suppose a medical procedure $p$ only requires two concurrent resources $r_{1}$ and $r_{2}$, the resource demand map is $\mathcal{M}_{r}=\left\{\left(p, r_{1} \otimes r_{2}\right)\right\}$. To model the concurrent resource demands, we build a statechart with two states ini and end. The statechart only contains one transition from state ini to state end with guard $V_{r_{1}} \& \& V_{r_{2}}$. The entry action of the state end assigns the corresponding procedure variable $V_{p}$ to be true. The statechart ensures that the procedure $p$ is able to be executed only when both $r_{1}$ and $r_{2}$ are available, i.e., $r_{1}$ and $r_{2}$ are concurrent resources. Fig. 8 depicts the concurrent resource statechart.

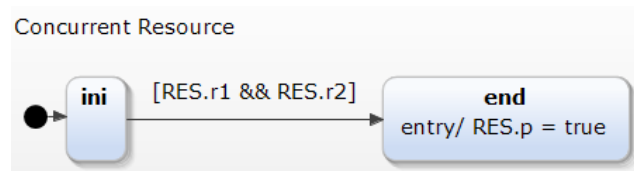

Fig. 8. Concurrent Resource Statechart

The alternative resource statechart is similar to the concurrent resource statechart except that the transition guard is $V_{r_{1}} \| V_{r_{2}}$, as shown in Fig. 9. The alternative resource statechart ensures that the procedure $p$ is able to be executed when either one of $r_{1}$ and $r_{2}$ is available, i.e., $r_{1}$ and $r_{2}$ are alternative resources.

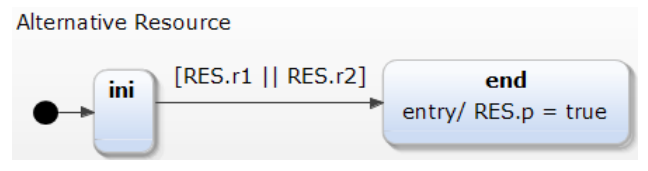

Fig. 9. Alternative Resource Statechart

Given a resource demand map $\mathcal{M}_{r}=\left\{\left(p, r_{1} \odot_{t_{0}} r_{2}\right)\right\}$, to model the sequence resource demands, we build a statechart with three states ini, tem, and end. The statechart contains two transitions: a transition from state ini to state tem with guard $V_{r_{1}}$ and a transition from state tem to state end with guard cur $\mathrm{T}-\mathrm{t}>=\mathrm{t}_{0} \& \& V_{r_{2}}$. In the transition guard, cur $\mathrm{T}$ is the current system time and $t$ is the time when the resource $r_{1}$ is available. The variable $t$ is assigned to the value of curT in the entry action of state tem. Similarly, the entry action of the state end assigns the corresponding procedure variable $V_{p}$ to be true. The statechart ensures that the procedure $p$ is able to be executed only when $r_{1}$ is available and $r_{2}$ is available after $t_{0}$ time units, i.e., $r_{1}$ and $r_{2}$ are sequence resources. Fig. 10 depicts the sequence resource statechart.

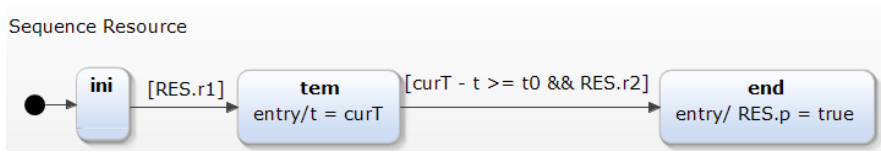

Fig. 10. Sequence Resource Statechart

Given a resource demand map $\mathcal{M}_{r}$, we model each element $(p, d) \in \mathcal{M}_{r}$ with a statechart by composing the three basic resource relationship statecharts shown in Fig. 8, Fig. 9, and Fig. 10 according to the resource demand $d$. Example 6 illustrates how to compose the basic resource relationship statecharts for the CT scan scenario.

Example 6: Given CT scan resource demand $d=$ CT_machine $\otimes$ CT_technician $\odot 10$ radiologist, we apply the concurrent resource statechart shown in Fig. 8 to the demand CT_machine $\otimes$ CT_technician and apply the sequence resource statechart shown in Fig. 10 to the rest part of $d$. Hence, the statechart contains three states ini, tem, and end. The entry actions of state tem and state end are $t=$ curT and CTscan $=$ true, respectively. There are two transitions in the statechart: a transition from state ini to state tem with guard machine\&\&technician and a transition from state tem to state end with guard $\operatorname{cur} \mathrm{T}-\mathrm{t}>=10 \& \&$ radiologist. The statechart resource model is shown in Fig. 11.

\section{Model Medical Resource Available Times}

In this section, we first extract and convert medical resource available times from given resource available time schedules into a map structure, and then based on the map structure, we design a statechart and a Java class to model resource available times. 


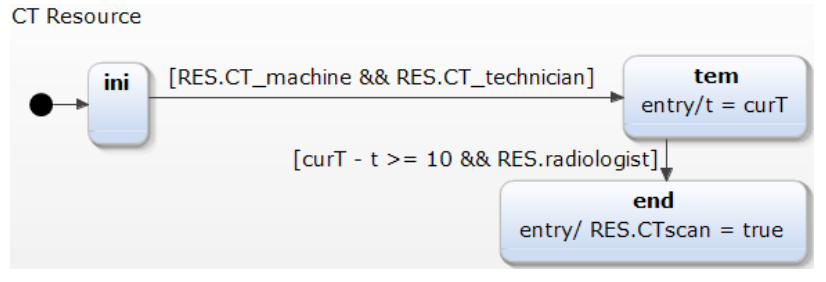

Fig. 11. CT Resource Model

\section{A. Map Structure}

In medical facilities, medical resource schedules are usually represented as time tables. For instance, Fig. 12 shows a schedule of four medical resources, i.e., Technologist, Nurse, Axis, and Meridian, which are required for the MSC (mesenchymal stromal cell) bone imaging procedure [25]. Note that the Waiting time is for patients that are not considered as medical resources in this paper. In Fig. 12, each time slot represents 10 minutes and are relative time. Medical resources are unavailable during the shaded time slots. For example, the Technologist is only available from time 0 to 20 minutes and from 35 to 185 minutes.

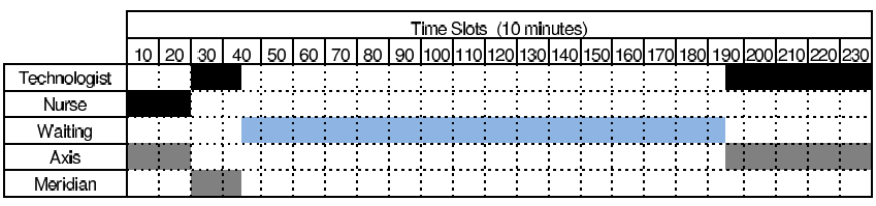

Fig. 12. Schedule for MSC Bone Imaging Resources [25]

To extract medical resource available times from given time tables, we define the time interval and medical resource available time map as follows.

Definition 8 (Time Interval): A time interval $T$ is represented as $\left[t_{s}, t_{e}\right]$, where $t_{s}$ and $t_{e}$ are the start and end time points of the time interval $T$, respectively.

In the resource available time map structure (key, $\overrightarrow{v a l u e})$, the key is medical resources required by medical guidelines. The value of the resource available time map is time intervals within which the corresponding resource (key) is available. As a medical resource may have multiple available time intervals, we use an array of all available times intervals to represent the value in the resource available time map structure. The formal definition of the resource available time map structure given below.

Definition 9 (Medical Resource Available Time Map): Given a set of medical resources $R=\left\{r_{1}, r_{2}, \ldots, r_{n}\right\}$ and a resource schedule $S$, the medical resource available time model $\mathcal{A}$ is defined as a map

$$
\begin{aligned}
\mathcal{M}_{a}= & \left\{\left(r_{1},\left\{T_{1}^{1}, T_{2}^{1}, \ldots, T_{m_{1}}^{1}\right\}\right),\right. \\
& \left(r_{2},\left\{T_{1}^{2}, T_{2}^{2}, \ldots, T_{m_{2}}^{2}\right\}\right), \\
& \ldots \ldots \ldots \\
& \left.\left(r_{n},\left\{T_{1}^{n}, T_{2}^{n}, \ldots, T_{m_{n}}^{n}\right\}\right)\right\},
\end{aligned}
$$

where $T_{i}^{j}$ is the $i$ th time interval within which the medical resource $r_{j}$ is available.
The following example shows how to obtain the resource available time map from the simplified CT scan scenario.

Example 7: The CT scan scenario requires CT machines, CT technicians, and radiologists. Fig. 13 shows an example schedule of CT scan resources in 40 minutes. Each time slot represents 5 minutes and are relative time. The shaded time slots are unavailable for corresponding resources. In Fig. 13, the $C T$ Machine has two available time intervals $[10,25]$ and $[35,40]$. Similarly, we can identify the available time intervals for the other two resources. The medical resource available time model of the CT scan example is

$$
\begin{aligned}
\mathcal{M}_{\mathrm{CT}}=\{ & (\text { CT_machine },\{[10,25],[35,40]\}), \\
& (\text { CT_technician, }\{[0,10],[15,25],[35,40]\}), \\
& (\text { radiologist },\{[0,15],[30,40]\})\} .
\end{aligned}
$$

\begin{tabular}{|l|c|c|c|c|c|c|c|c|}
\cline { 2 - 9 } \multicolumn{1}{c|}{} & \multicolumn{7}{c|}{ Time Slots (5 minutes) } \\
\cline { 2 - 9 } \multicolumn{1}{c|}{} & 5 & 10 & 15 & 20 & 25 & 30 & 35 & 40 \\
\hline CT Machine & & & & & & & & \\
\hline CT Technician & & & & & & & & \\
\hline Radiologist & & & & & & & & \\
\hline
\end{tabular}

Fig. 13. Schedule for CT Scan Resources

\section{B. Statechart Model}

Given a medical resource available time map $\mathcal{M}_{a}=$ $\{(r,\{T\})\}$, we develop a procedure to represent $\mathcal{M}_{a}$ with statecharts in three steps: (1) use a configuration file to store resource available times specified in $\mathcal{M}_{a}$; (2) implement an external Java class ResAva, which is supported by Yakindu statecharts, to read the resource available time configuration file and check whether resources are available at current system time; and (3) design a statechart to access the Java class ResAva and represent medical resource available times. The approach has two advantages: (1) when medical resource available times change which can happen quite often, we only need to update the resource available time configuration file without the need for statechart model changes; (2) as there is only one statechart model for resource available times, the workload for validating and verifying the correctness of resource available time model is reduced.

In the resource available time configuration file, each element in the resource available time map $\mathcal{M}_{a}$ is stored in one line. For each map element, we use colon (:) and semicolon (;) to separate the resource $r$ and its available time intervals $\{T\}$ and each available time interval $T$, respectively. For example, the resource available time configuration file of the CT scan scenario in formula (9) is shown in Fig. 14.

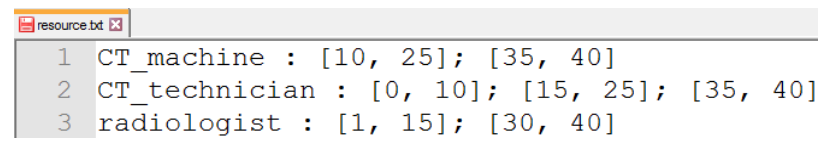

Fig. 14. Resource Available Time Configuration File of CT Scan Scenario

Yakindu statechart supports external Java code, we implement a Java class ResAva shown in Fig. 15 to read medical 
resource available time configuration files and check resource availabilities. The ResAva class provides two functions:

1) read(String resAvaPath) reads the medical resource available time configuration file located in resAvaPath;

2) check(long t,String res) checks whether the medical resource res is available at time $t$.

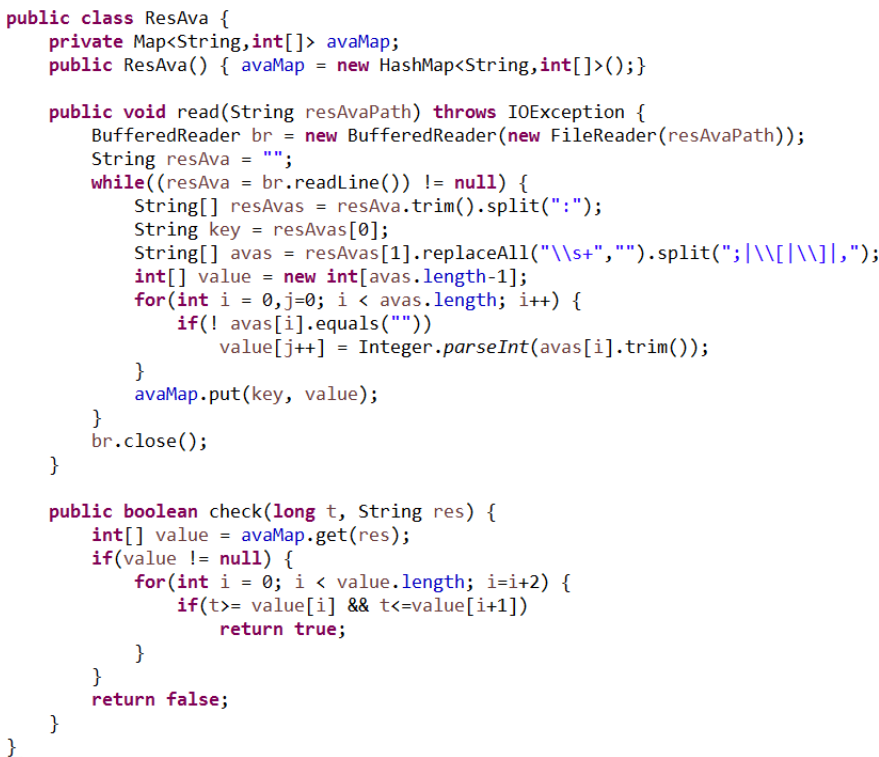

Fig. 15. ResAva Java Class

To model medical resource available times, we build a statechart with only one state that has a self-loop transition with guard true. The transition ensures that resource available times are checked at each statechart execution cycle. The entry actions of the state call $\operatorname{read}()$ function to read the configuration file and $\operatorname{check}()$ function for each resource $r$ in the given resource available time map $\mathcal{M}_{a}$ to check resource availabilities. We use Example 8 to show how to represent resource available time for the CT scan scenario.

Example 8: Given the resource available time map $\mathcal{M}_{a}$ of the CT scan scenario in formula (9), the corresponding resource availability configuration file is shown in Fig. 14.

To represent resource available times, we build the Resource statechart with only one state named Res which has a self-loop transition with guard true. As the given resource available time map $\mathcal{M}_{a}$ contains three medical resources CT_machine, CT_technician, and radiologist, we add four entry actions to the state Res:

1) ResAva.read("/resource.txt" reads the resource availability configuration file shown in Fig. 14;

2) RES.CT_machine = ResAva.check(curT, "CT_machine") checks current availability of the resource CT_machine;

3) RES.CT_technician = ResAva.check(curT, "CT_technician") checks current availability of the resource CT_technician;

4) RES.radiologist = ResAva.check(curT, "radiologist") checks current availability of the resource radiologist. The resource statechart is shown in Fig. 16.

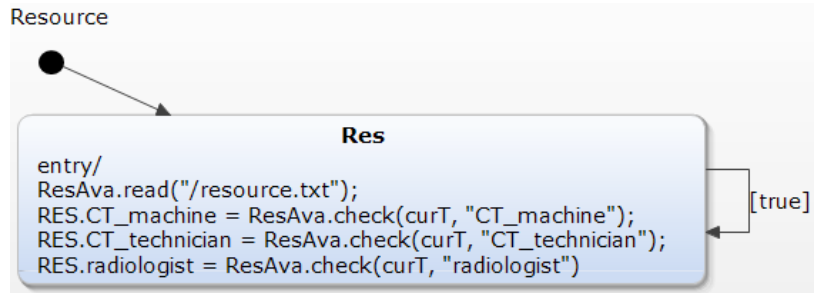

Fig. 16. Resource Statechart of CT Scan Scenario

\section{Integrate Medical Resource Models with Medical Guideline Statecharts}

To clinically validate and formally verify the safety of medical guideline models with consideration of medical resource available times and their relationships, we need to integrate medical resource models with medical guideline statecharts.

According to the medical resource modeling approach presented in Section $\mathrm{V}$, for each medical procedure $p$ in the given resource demand map, a boolean variable $V_{p}$ is declared to denote whether the procedure $p$ is able to execute. We use the declared procedure variable $V_{r}$ as an anchor to bridge the communication between medical resource models and medical guideline statecharts and modify medical guideline statecharts with following integration rules.

- Integration Rule 1: For each transition $T$ with guard $G$, if it is annotated with "//@RES : $\left.\left(p_{1}, d_{1}\right),\left(p_{2}, d_{2}\right), \ldots,\left(p_{n}, d_{n}\right)\right)$, the guard $G$ is replaced with $G=G \& \& V_{p_{1}} \& \& V_{p_{2}} \& \& \ldots \& \& V_{p_{n}}$;

- Integration Rule 2: For each state $S$, if it is annotated with "//@RES : $\left(p_{1}, d_{1}\right),\left(p_{2}, d_{2}\right), \ldots,\left(p_{n}, d_{n}\right)$ ", apply Integration Rule 1 to all incoming transitions of the state $S$ with new guards.

We also design Algorithm 2 to automatically integrate medical resource models with medical guideline statecharts. Example 9 illustrates how we apply the integration rules to integrate the resource demand given in formula (6) with the simplified stroke statechart.

Example 9: We integrate the resource models in Fig. 11 with the annotated stroke statechart model in Fig. 5. The transition $T_{1}$ from state tPAcheck to state tPA is annotated with "//@RES : (givetPA, tPA $\otimes$ nurse)" and has guard $G_{1}=$ tPAad. Based on Integration Rule 1, the transition $T_{1}$ ' guard is set as $G_{1}=$ tPAad \&\& RES.givetPA. The state CT is annotated by "//@RES : (CTscan, CT_machine $\otimes$ CT_technician $\odot_{10}$ radiologist)" and only has one incoming transition $T_{2}$ with guard $G_{2}=$ orderCT from state NeuAss. According to Integration Rule 2, we apply Integration Rule 1 to the transition $T_{2}$ and set the guard as $G_{2}=$ orderCT \&\& RES.CTscan. Fig. 17 shows the integrated stroke statechart, where the modified transitions are marked by red rectangles.

\section{Simplified Stroke Case Study}

In this section, we use the stroke scenario to illustrate the effectiveness and advantages of the proposed SMJV architecture 

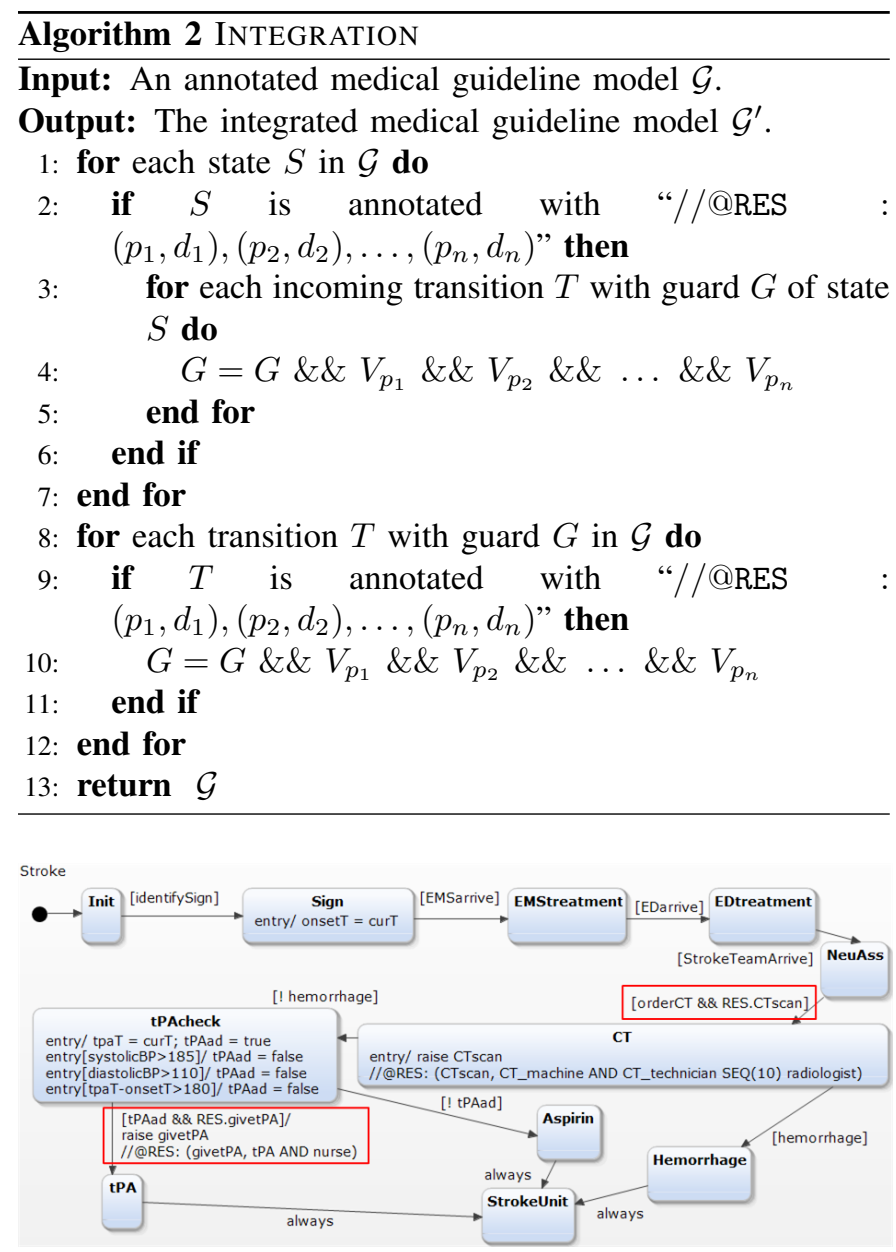

Fig. 17. Integrated Statechart of Simplified Stroke Scenario

which separately models medical resource available times and relationships and jointly verifies existing medical best practice guideline statecharts with resource models being integrated in.

\section{A. SMJV Architecture Validation}

The stroke statechart model given in Fig. 3 has only focused on the CT scan and tPA administration procedures, but omitted the details of other medical procedures. To validate the effectiveness of the proposed architecture, we extend the simplified stroke model by considering following scenarios with different patient conditions: (1) if a patient's blood pressure is not within the range required by tPA administration, a blood pressure control procedure needs to be performed; (2) if tPA administration is approved within 3 hours from onset of stroke symptoms, an IV tPA procedure is performed; (3) if tPA administration is approved in the 3-6 hour window from the onset time, an IA tPA procedure is performed; and (4) if tPA is not approved, aspirin is given to patients.

We use the proposed approach to annotate resource demand, model resource relationships and available times, and integrate resource models with the extended stroke statechart model. The resource models and integrated stroke statechart are shown in Fig. 18 and Fig. 19, respectively.

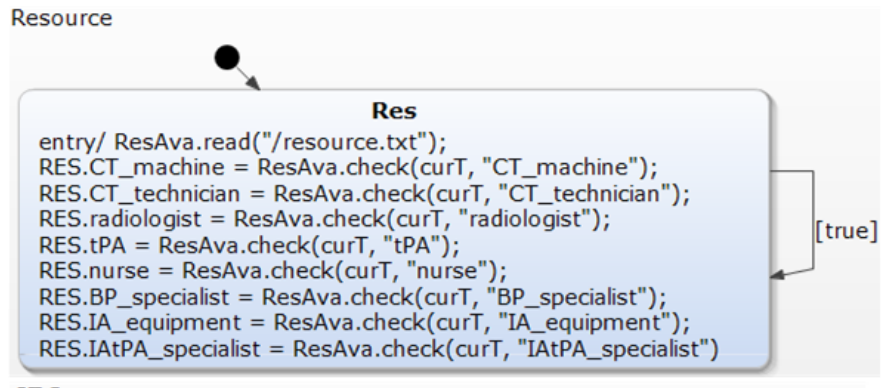

CT Resource

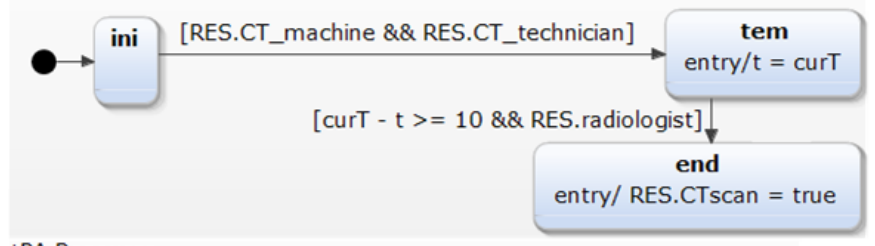

tPA Resource

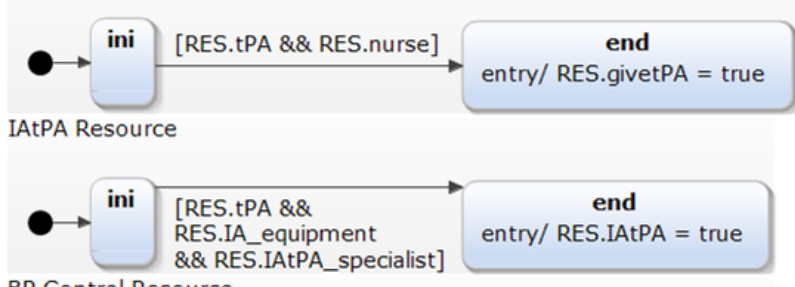

BP Control Resource

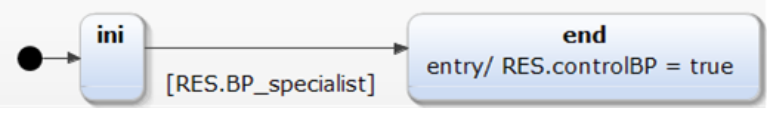

Fig. 18. Stroke Resource Model

To clinically validate and formally verify the safety of the stroke statechart with the consideration of resource available times and relationships, we run simulations of the integrated stroke statechart model (Fig. 19) through Yakindu, transform the integrated stroke model to an UPPAAL model with the Y2U tool [12] (Fig. 20), and verify the safety properties in UPPAAL.

In addition to the properties $\mathbf{P 1}$ and $\mathbf{P 2}$ given in formula (1) and formula (2), we also need to verify property $\mathbf{P 3}$ that the IA tPA administration must be completed within 6 hours from onset of stroke symptoms, i.e.,

$$
A[\text { ] Stroke.IAtPA imply tpaT - onsetT }<=360
$$

Assume a patient's onset time of stroke symptom is 0 , the resource schedule is given in Fig. 21. Both simulation and verification results show that the safety property P1 and P3 hold, but $\mathbf{P 2}$ fails.

The case study demonstrates the following: (1) the proposed separately model and jointly verify approach are able to capture unsafety properties caused by temporarily unavailable resources in both clinical validation and formal verification processes; and (2) the approach can address patient care safety under different patient conditions by exploring different execution paths in medical guideline models with correspond- 


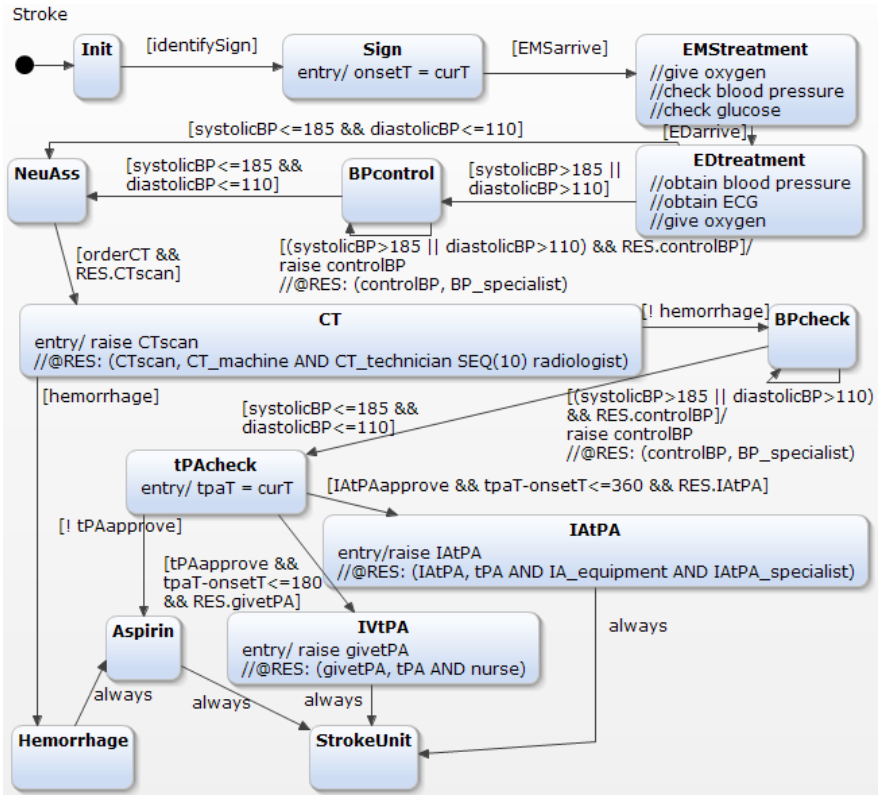

Fig. 19. Integrated Stroke Statechart

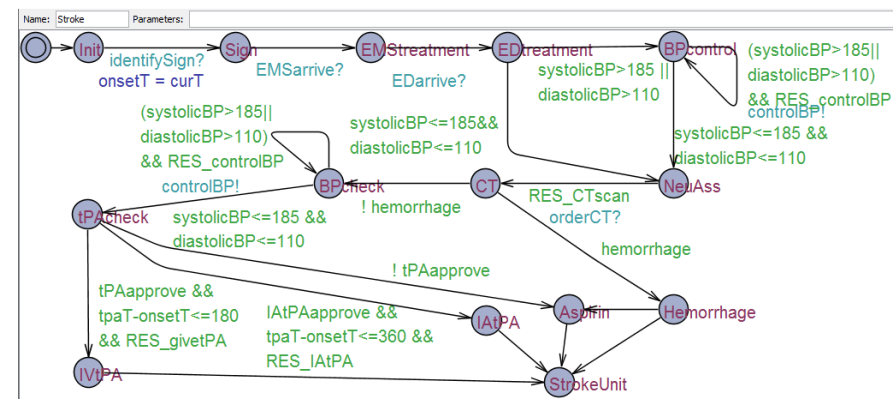

Fig. 20. Stroke UPPAAL Model

ing resource available times and their relationships being integrated in.

\section{B. Advantages of the SMJV Architecture}

The proposed SMJV architecture uses separate statecharts to model medical resource available times and relationships and integrates resources models with medical guideline statecharts to clinically validate and formally verify safety properties in the presence of resource demands and potential unexpected resource delays. An alternative approach to address the medical resource demand issue in existing medical guideline statecharts is to directly add medical resource demands as transition guards or state constraints. We call this method as direct modification approach. Compared with the direct modification approach, the proposed SMJV architecture has two major advantages. First, the separated resource demand statecharts and resource available time statecharts of common medical procedures can be reused by multiple medical guideline models. Second, the proposed approach requires minimal model modifications to accommodate frequent resource available time changes in emergency care environment. We

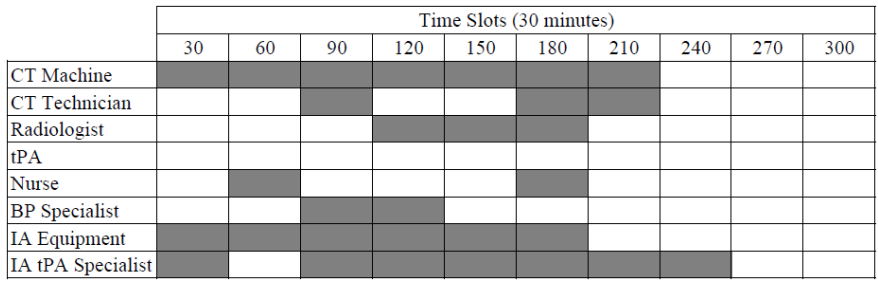

Fig. 21. Stroke Resource Schedule

use the CT scan scenario in the stroke care to show the two advantages.

Assume a resource available time schedule is given in Fig. 21. The direct modification approach models CT scan resource demands by modifying the guard of transition from state NeuAss to state CT to be orderCT\&\&CT_machine $>=210 \& \& C T$ techinician $>=$ $210 \& \&$ radiologist $>=180$. With the proposed approach, the CT scan resource demands are modeled with two statecharts as depicted by Fig. 11 and Fig. 16 and a resource available time configuration file shown in Fig. 14. The resource models are integrated with the stroke guideline statecharts by changing the guard of transition from state NeuAss to state CT from orderCT to orderCT\&\&CT_scan.

On the surface, it seems that the SMJV requires more work than the direct modification approach. However, once the resource demand model for CT scan is developed, it can be reused by other medical guideline models that require CT scan in their patient care, such as in the guideline model for acute appendicitis [10]. Even within the same medical guideline model, a medical procedure may be required at different patient care stages. For instance, in the stroke guideline model, there are two places that require the blood pressure control procedure as shown in Fig. 19, one when a patient arrives the emergency department, and the other before tPA is administrated. But with the direct modification approach, for every medical guideline models where CT scan is needed, the corresponding transition guards has to be modified by adding the conjunction condition orderCT\&\&CT_machine $>=210 \& \&$ CT_techinician $>=$ 210\&\&radiologist $>=180$.

Furthermore, if the CT machine's available time changes from $[210,300]$ to $[220,300]$, with the proposed approach, we only need to update the resource available time configuration file shown in Fig. 14. As none of the statechart models are changed, medical professionals only need to re-validate the resource available time configuration file and run our system to verify safety properties with updated resource available times. However, for the same available time change, the direct modification approach would need to modify all the transition guards leading to the CT scan. As the stroke statechart is modified to reflect updated resource availability, medical professionals are required to re-validate the stroke guideline model. For formal verification, the statechart needs to be retransformed to UPPAAL timed automata to re-verify the safety 
properties.

\section{CONCLUSION}

The paper presents an approach to annotate resource demands in existing medical guideline models, separately model medical resource available times and relationships with statecharts, and integrate resources models with executable medical guideline statecharts. The proposed architecture can be easily integrated into our existing platform [12] and automatically transform medical guideline statecharts with resource demands to UPPAAL timed automata so that medical guideline safety properties in the presence of temporarily unavailable medical resources can be formally verified.

We have use a simplified stroke scenario as a case study to investigate the effectiveness and validity of the SMJV architecture. The case study demonstrates: (1) the SMJV architecture allows different domain personals to make independent model modifications on resource available times as well as on medical guideline models, and requires minimal changes to integrate resource available time and relationship considerations into existing medical guideline models; (2) the SMJV architecture is able to identify unsafe properties caused by temporarily unavailable medical resources, and is easy adapt to frequent and unexpected resource available time changes; and (3) the separately modeled resource statecharts can be reused by multiple procedures within a medical guideline model and also by multiple medical guideline models.

\section{REFERENCES}

[1] R. Alur and D. L. Dill. A theory of timed automata. Theoretical computer science, 126(2):183-235, 1994.

[2] A. S. Association. Ischemic stroke. https://www.strokeassociation.org/ idc/groups/stroke-public/@wcm/@hcm/documents/downloadable/ucm _ 309725.pdf.

[3] A. S. Association. Stroke treatment. http://www.strokeassociation. org/STROKEORG/AboutStroke/Treatment/Stroke-Treatment_UCM_ 492017_SubHomePage.jsp.

[4] M. Balser, C. Duelli, and W. Reif. Formal semantics of asbru an overview. Proc. of the 6th Biennial World Conference on Integrated Design and Process Technology, 5(5):1-8, 2002.

[5] G. Behrmann, A. David, and K. Larsen. A tutorial on uppaal. In Formal Methods for the Design of Real-Time Systems, pages 200-236. Springer, 2004.

[6] T. Cayirli and E. Veral. Outpatient scheduling in health care: a review of literature. Production and operations management, 12(4):519-549, 2003.

[7] S. Christov, B. Chen, G. S. Avrunin, L. A. Clarke, L. J. Osterweil, D. Brown, L. Cassells, and W. Mertens. Formally defining medical processes. Methods of Information in Medicine, 47(5):392-398, 2008.

[8] S. Christov, B. Chen, G. S. Avrunin, L. A. Clarke, L. J. Osterweil, D. Brown, L. Cassells, and W. Mertens. Rigorously Defining and Analyzing Medical Processes: An Experience Report, pages 118-131. Springer Berlin Heidelberg, Berlin, Heidelberg, 2008.

[9] D. Conforti, F. Guerriero, and R. Guido. Optimization models for radiotherapy patient scheduling. 4OR: A Quarterly Journal of Operations Research, 6(3):263-278, 2008.

[10] S. Di Saverio, A. Birindelli, M. D. Kelly, F. Catena, D. G. Weber, M. Sartelli, M. Sugrue, M. De Moya, C. A. Gomes, A. Bhangu, F. Agresta, E. E. Moore, K. Soreide, E. Griffiths, S. De Castro, J. Kashuk, Y. Kluger, A. Leppaniemi, L. Ansaloni, M. Andersson, F. Coccolini, R. Coimbra, K. S. Gurusamy, F. C. Campanile, W. Biffl, O. Chiara, F. Moore, A. B. Peitzman, G. P. Fraga, D. Costa, R. V. Maier, S. Rizoli, Z. J. Balogh, C. Bendinelli, R. Cirocchi, V. Tonini, A. Piccinini, G. Tugnoli, E. Jovine, R. Persiani, A. Biondi, T. Scalea, P. Stahel, R. Ivatury, G. Velmahos, and R. Andersson. Wses jerusalem guidelines for diagnosis and treatment of acute appendicitis. World Journal of Emergency Surgery, 11(1):34, Jul 2016.

[11] J. Fox, N. Johns, and A. Rahmanzadeh. Disseminating medical knowledge: the proforma approach. Artificial Intelligence in Medicine, 14(12): 157 - 182, 1998.

[12] C. Guo, S. Ren, Y. Jiang, P.-L. Wu, L. Sha, and R. Berlin. Transforming medical best practice guidelines to executable and verifiable statechart models. In 2016 ACM/IEEE 7th International Conference on CyberPhysical Systems (ICCPS), pages 1-10, April 2016.

[13] D. Gupta and B. Denton. Appointment scheduling in health care: Challenges and opportunities. IIE transactions, 40(9):800-819, 2008.

[14] R. Hall and J. Partyka. Handbook of healthcare system scheduling. Springer, 2012.

[15] D. Harel. Statecharts: A visual formalism for complex systems. Science of computer programming, 8(3):231-274, 1987.

[16] Itemis. Yakindu statechart tools (sct). https://www.itemis.com/en/ yakindu/statechart-tools/, July 2016.

[17] E. C. Jauch, B. Cucchiara, O. Adeoye, W. Meurer, J. Brice, Y. Y.-F. Chan, N. Gentile, and M. F. Hazinski. Part 11: adult stroke: 2010 american heart association guidelines for cardiopulmonary resuscitation and emergency cardiovascular care. Circulation, 122(18 suppl 3):S818S828, 2010.

[18] J. Kim, I. Kang, J. Y. Choi, and I. Lee. Timed and resource-oriented statecharts for embedded software. IEEE Transactions on Industrial Informatics, 6(4):568-578, Nov 2010.

[19] M. Liu and W. Su. An improvement of scheduling method for medical resource order and distribution. In 35th Chinese Control Conference (CCC), July 2016.

[20] D. I. McIsaac, K. Abdulla, H. Yang, S. Sundaresan, P. Doering, S. G. Vaswani, K. Thavorn, MPharm, and A. J. Forster. Association of delay of urgent or emergency surgery with mortality and use of health care resources: a propensity scorematched observational cohort study. Canadian Medical Association Journal, 189(27), 2017.

[21] B. A. McKinley, L. J. Moore, J. F. Sucher, S. R. Todd, K. L. Turner, A. Valdivia, R. M. Sailors, and F. A. Moore. Computer protocol facilitates evidence-based care of sepsis in the surgical intensive care unit. Journal of Trauma and Acute Care Surgery, 70(5):1153-1167, 2011.

[22] A. C. of Surgeons. Committee on Trauma. ATLS advanced trauma life support program for doctors. American College of Surgeons, 2004.

[23] V. L. Patel, V. G. Allen, J. F. Arocha, and E. H. Shortliffe. Representing clinical guidelines in glif. Journal of the American Medical Informatics Association, 5(5):467-483, 1998.

[24] J. Patrick and M. L. Puterman. Improving resource utilization for diagnostic services through flexible inpatient scheduling: A method for improving resource utilization. Journal of the Operational Research Society, 58(2):235-245, 2007.

[25] E. Prez, L. Ntaimo, W. E. Wilhelm, C. Bailey, and P. McCormack. Patient and resource scheduling of multi-step medical procedures in nuclear medicine. IIE Transactions on Healthcare Systems Engineering, 1(3):168-184, 2011

[26] E. A. Prince, S. H. Ahn, and G. M. Soares. Intra-arterial stroke management. Seminars in interventional radiology, 30(03):282-287, 2013.

[27] P. Terenziani, S. Montani, A. Bottrighi, M. Torchio, G. Molino, and G. Correndo. The glare approach to clinical guidelines: main features. Stud. Health Technol. Inform., pages 162-166, 2004.

[28] S. W. Tu and M. A. Musen. Modeling data and knowledge in the eon guideline architecture. Medinfo, pages 280-284, 2001.

[29] I. B. Vermeulen, S. M. Bohte, S. G. Elkhuizen, H. Lameris, P. J. Bakker, and H. La Poutré. Adaptive resource allocation for efficient patient scheduling. Artificial intelligence in medicine, 46(1):67-80, 2009.

[30] P. Wu, D. Raguraman, L. Sha, R. Berlin, and J. Goldman. A treatment validation protocol for cyber-physical-human medical systems. In Software Engineering and Advanced Applications (SEAA), 2014 40th EUROMICRO Conference on, pages 183-190, Aug 2014.

[31] P. Wu, L. Sha, R. B. Berlin, and J. M. Goldman. Safe workflow adaptation and validation protocol for medical cyber-physical systems. In 2015 41st Euromicro Conference on Software Engineering and Advanced Applications, pages 464-471, Aug 2015. 